\title{
Relação do traumatismo cranioencefálico grave com o tempo de permanência na ventilação mecânica invasiva
}

\author{
Relationship of serious cranioencephalic traumatism with the permanence time in invasive \\ mechanical ventilation
}

\section{Relación del traumatismo cranioencefálico grave con el tiempo en la ventilación mecánica invasiva}

Isabela Kerber Alves ${ }^{1 *}$, Aline Arcari Santos ${ }^{1}$, Tatielly Ricarte Sousa ${ }^{1}$, Neide Garcia Ribeiro Castilho ${ }^{1}$, Tieverton Guilherme de Oliveira Santos ${ }^{1}$, Nicole Lima ${ }^{1}$, Larissa Karen Pereira Santos ${ }^{1}$, Laurijane Santos Do Nascimento ${ }^{1}$, Yargo Alexandre de Farias Machadoํㅗ․, Egberto Luiz Felício Junior ${ }^{1}$.

\section{RESUMO}

Objetivo: Descrever a relação do trauma cranioencefálico grave com o tempo de permanência na VMI. Métodos: Trata-se de uma pesquisa de campo com característica quantitativo-descritivo desenvolvida no período de maio a outubro de 2020, em pacientes atendidos no Hospital de Urgência e Emergência do estado de Rondônia, a partir de coleta documental em prontuários médicos de vítimas de TCE grave de ambos os gêneros e com faixa etária acima de 18 anos de idade. Resultados: Obteve-se um total de 41 prontuários médicos, com idade média dos pacientes de $37,29 \pm 14,70$, sendo a maioria homens (32), com prevalência de acidentes com motocicleta (24 casos, $58,54 \%$ ). Quanto ao tempo de permanência na VMI constatou-se que o tempo médio foi de $9,70 \pm 8,25$ dias. Verificou-se que os pacientes que foram à óbito demonstraram um tempo menor de permanência na VMI comparado com os que sobreviveram (12,8 $\pm 10,0$ dias). Conclusão: Referente ao tempo de permanência da VMI, houve variação de dias entre pacientes com maior e menor pontuação da escala de coma de Glasgow, onde os que tiveram menor pontuação são os que em maioria permaneceram por menos tempo na ventilação, sendo indivíduos com maior gravidade do estado clínico geral.

Palavras-chave: Lesões encefálicas traumáticas, Respiração artificial, Emergência, Unidades de terapia intensiva.

\begin{abstract}
Objective: To describe the relationship between severe traumatic brain injury and the time permanence on IMV. Methods: This is field research with a quantitative-descriptive characteristic developed from May to October 2020, in patients treated at the Hospital of Urgency and Emergency in the state of Rondônia, based on documentary collection in medical records of the death of severe TBI of both genders and with an age range above 18 years of age. Results: A total of 41 medical records were obtained, with an average age of patients $37.29 \pm 14.70$, with the majority of men (32), with a prevalence of motorcycle accidents (24 cases, $58.54 \%$ ), referring to when the time spent in the IMV, it was found that the average time was $9.70 \pm 8.25$ days. It was found that patients who died demonstrated a shorter duration of stay on IMV compared to those who survived (12.8 \pm 10.0 days). Conclusion: Regarding the duration of IMV stay, there was a variation of days between patients with the highest and lowest score on the Glasgow coma scale, where those with the lowest score are those who mostly remained for less time on ventilation, being individuals with greater severity of general medical condition.
\end{abstract}

Keywords: Brain injuries traumatic, Respiration artificial, Emergencies, Intensive care units.

\section{RESUMEN}

Objetivo: Describir la relación entre el traumatismo craneoencefálico grave y el tiempo de permanencia en VMI. Métodos: Se trata de una pesquisa de campo con caracteristica quantitativo-descritivo desenvolvida en el periodo de mayo a octubre de 2020, en pacientes atendidos en el Hospital de Urgencia e Emergencia, en el estado de Rondônia desde la coleta documental en prontuarios medicos de víctimas de TCE grave de ambos los generos y con idad en media de 18 años. Resultados: Se obtuvo un total de 41 historias clinicas,

${ }^{1}$ Complexo Hospitalar Regional de Cacoal (COHREC), Cacoal - RO. *E-mail: isabelakerber@outlook.com 
con una edad promedio de 37,29 \pm 14,70 años, la mayoría hombres (32), con una prevalencia de accidentes de motocicleta ( 24 casos, $58,54 \%$ ), referida al tiempo de estancia en En la VMI se encontró que el tiempo promedio fue de $9,70 \pm 8,25$ días. Se encontró que los pacientes que fallecieron demostraron un menor tiempo de permanencia en VMI en comparación con los que sobrevivieron (12,8 $\pm 10,0$ días). Conclusión: En cuanto a la duración de la estancia en VMI, hubo variación de días entre los pacientes con mayor y menor puntaje en la escala de coma de Glasgow, donde los que menor puntaje son los que mayoritariamente permanecieron menos tiempo en ventilación, siendo los individuos con mayor severidad de condición médica general.

Palabras clave: Lesiones traumáticas del encéfalo, Respiración artificial, Urgências médicas, Unidades de cuidados intensivos.

\section{INTRODUÇÃO}

Define-se como TCE uma lesão primária contusa induzida por força mecânica e, geralmente apresenta heterogeneidade quanto aos fatores etiológicos (acidentes automobilísticos e ciclísticos, atropelamentos, quedas (de altura e da própria altura), lesões por arma de fogo e arma branca, agressões físicas, dentre outras), mecanismo, gravidade, patologia e prognóstico com resultados amplamente variados (JOVANOVIC B, et al., 2016; MAAS A, 2016).

É uma condição crônica com efeitos multissistêmicos que pode promover interrupção da função cerebral temporária ou permanente, uma lesão de crânio ou do cérebro que seja suficientemente grave para comprometer a função normal dessa estrutura, podendo causar déficits físicos, psicossociais e sociais importantes em até $60 \%$ dos casos, prejudicando não só a vida do paciente, como toda a sociedade que interferem nos dados demográficos (HAMEL RN e SMOLIGA JJ, 2019; KHELLAF A, et al., 2019; SILVA PE, et al., 2018; PONTE FR, et al., 2017) .

Segundo Vella MA, et al. (2018) o TCE pode ser clinicamente estratificado em leve (10\%), moderado $(60 \%)$ e grave $(100 \%)$ a partir da Escala de Coma de Glasgow, considerando taxas de incapacidade permanente associada de 10,60 e 100\%, respectivamente e, as taxas de mortalidade geral atingem 20 $30 \%$. Khellaf A, et al. (2019) descreve que a gravidade clínica dessa doença pode ser estratificada em função do escore da Escala de Coma de Glasgow da seguinte maneira: leve (14-15 pontos), moderado (913 pontos) e grave (3-8 pontos).

Uma vez que o cérebro é responsável por diversas funções (habilidades cognitivas, pensamento, comportamento, habilidades motoras, entre outras), os impactos gerados pelo TCE são devastadores (REIS HFC, et al., 2012). Desse modo, os sobreviventes dessa doença podem enfrentar uma série de deficiências, como: intolerância à exercício físico, mudanças emocionais e comportamentais, fadiga e insônia, entre outras (HAMEL RN e SMOLIGA JJ, 2019).

Segundo Jovanovic B, et al. (2016), Ponte FR, et al. (2017) e Picetti E, et al. (2020) o TCE é o principal determinante de morbidade, incapacidade e/ou invalidez e mortalidade, sendo reconhecido como um grave problema de saúde pública em todo o mundo, especialmente entre as pessoas com faixa etária produtiva e sobretudo, é a principal causa de óbito entre as pessoas com predomínio no gênero masculino. Os piores resultados constatados em pacientes mais velhos sugerem a condição clínica do TCE (YOU W, et al., 2016)

O TCE é a maior causa de internações em Unidades de Terapia Intensiva (UTIs), já submetidos a IOT e a VMI que promove boa condição ventilatória na fase aguda do trauma e, sobretudo, diminui o sofrimento encefálico e interfere na geração de complicações secundárias. Enfatiza-se que o TCE se associa a lesões em outras regiões do corpo (politraumatismo) e, consequentemente necessita de períodos prolongados de recuperação com uso de ventilação mecânica invasiva (VMI), que inevitavelmente influencia no aparecimento e aumento na incidência de complicações importantes intra-hospitalares, pois o tratamento requer o uso de terapia neuroprotetora com sedação profunda e repouso prolongado no leito e, isso pode gerar uma importante fraqueza adquirida na UTI resultando em mau prognóstico funcional (JOVANOVIC B, et al., 2016; PONTE FR, et al., 2017; REIS HFC, et al., 2012; PICETTI E, et al., 2020 e SILVA PE, et al., 2018). 
De acordo com Protheroe RT e Gwinnutt CL (2011); Haddad SH e Arabi YM (2012) o TCE pode ser dividido em dois grupos distintos, a) lesão cerebral primária - decorre do evento traumático devido ao cisalhamento e compressão do tecido cerebral circundante e, promove dano físico ao parênquima (células gliais, tecidos e vasos) que não é passível de alteração e não pode ser revertida e, b) lesão cerebral secundária - deriva de insultos intracranianos (edema cerebral, hematomas, hipertensão intracraniana, infecção e convulsões dentre outros), extracranianos e/ou sistêmicos (hipotensão e hipertensão arterial sistêmica, hipoxemia, hipercapnia, febre, anemia, entre outros) que resulta e complica nas primeiras horas e dias seguintes da lesão e, costuma ser passível de prevenção e reversão (BOHMAN LE, et al., 2011).

O volume do compartimento intracraniano é composto de tecido cerebral (83\%), líquido cefalorraquidiano (11\%), sangue venoso e arterial (6\%), cada um desses elementos apresenta uma interdependência para manter um ambiente homeostático no interior da calota craniana. Assim, o fluxo sanguíneo cerebral é mantido constante em condições normais por meio de mecanismos autorregulatórios cerebrais em uma faixa de pressão arterial. Ao aumentar um compartimento, por um hematoma, por exemplo, ocorre uma cascata de mecanismos compensatórios, afim de prevenir a hipertensão intracraniana (GALGANO M, et al., 2017).

Há um amplo consenso de que o manejo adequado se concentra em estabelecer e manter as vias aéreas desobstruídas com uso da intubação orotraqueal (IOT), empregando analgesia e sedação adequadas para permitir a prevenção de hipóxia, hipercapnia ou hipocapnia, coagulopatia, hipotermia, hipotensão e controle da hemorragia (PROTHEROE RT e GWINNUTT CL, 2011), os quais representam critérios terapêuticos pré-clínicos que podem favorecer na melhora e também na sobrevida do paciente com TCE grave, evitando o aumento da gravidade das lesões extracerebrais (BOER C, et al., 2012; TRIMMEL H, et al., 2017).

Segundo Haddad SH e Arabi YM (2012); Reis HFC, et al. (2013); Schimer-Mikalsen K, et al. (2015) o principal objetivo do tratamento é impedir o desenvolvimento da lesão cerebral secundária a partir da elevação da pressão intracraniana, garantindo dessa forma a circulação, oxigenação e perfusão adequadas para o cérebro (2012). Reconhece-se que o TCE não deve ser pensado como um evento agudo, mas como um gatilho de lesão progressiva que pode ocorrer ao longo de horas, dias, semanas, meses e até anos (MAAS A, 2016). Os pacientes que apresentam lesões graves, como é o caso de TCE, apresentam alto risco de adquirir infecção nosocomial, especialmente a pneumonia associação à ventilação mecânica invasiva (PAVM) (JOVANOVIC B, et al., 2015).

Sendo assim, o presente estudo teve como objetivo descrever a relação do trauma cranioencefálico grave com o tempo de permanência na VMI em pacientes atendidos em um Hospital de Urgência e Emergência no estado de Rondônia.

\section{MÉTODOS}

O presente estudo trata-se de uma pesquisa de campo que tem como objetivo conseguir informações e/ou conhecimentos sobre um problema, para o qual se investiga uma resposta, ou sobre uma hipótese, se queira alguma comprovação, ou, ainda, tem o propósito de descobrir novos fenômenos ou relações entre eles (MARCONI MA e LAKATOS EM, 2017).

Adicionalmente, consiste na observação de fatos e fenômenos tal como ocorrem (espontaneamente), na coleta de informações a eles referentes e no registro de variáveis que se presume importantes para interpretá-los. Além disso, condiz com uma coleta documental, que se refere com a coleta de dados apenas em documentos, escritos ou não, que constituem o que se denomina de fontes primárias, no momento da ocorrência do fato ou fenômeno, ou após. Caracteristicamente, é uma pesquisa quantitativo-descritivo, que consiste na investigação de pesquisa empírica, com finalidade de delinear ou analisar as características de fatos ou fenômenos (MARCONI MA e LAKATOS EM, 2017).

Desse modo, procedeu-se na coleta de dados de prontuários médicos, realizado em um Hospital de Urgência e Emergência do estado de Rondônia, consistindo em referência de urgência e emergência da Amazônia Legal. 
Para a coleta dos dados considerou-se os seguintes critérios de inclusão: vítimas de TCE com classificação grave pela Escala de Coma de Glasgow com pontuação de 3-8 pontos, conforme proposto por Haddad SH e Arabi YM (2012); Khellaf A, et al. (2019), que deram entrada no Hospital supracitado e necessitaram de $\mathrm{VMI}$ até o processo de extubação, independentemente do gênero e com faixa etária acima de 18 anos de idade.

Essa coleta procedeu-se no período de maio a outubro de 2020, onde os pesquisadores realizaram visitas diárias na Sala Vermelha e também na UTI do Hospital de Urgência e Emergência em dois períodos distintos (matutino e vespertino).

Em contrapartida, os critérios de exclusão foram: vítimas com TCE leve e moderado, de acordo com a classificação anteriormente mencionada, vítimas de TCE com faixa etária inferior a 18 anos de idade, pacientes que não necessitaram de VMI, os que foram transferidos para outras entidades de assistência à saúde e aqueles que foram a óbito logo após a admissão (imediatamente).

Os dados foram coletados e inseridos diretamente no programa Microsoft Office Excel 2013 e, em seguida realizou-se a tabulação dos dados com análise estatística com o programa Statistical Package for the Social Sciences (SPSS) versão 20.0 e, realizou-se a descrição dos mesmos. Enfatiza-se que o presente estudo cumpriu com as prerrogativas e recomendações dispostas na Resolução no. 466, de 12 de dezembro de 2012 que diz respeito às Normas de Pesquisa Envolvendo Seres Humanos do Conselho Nacional de Saúde (CONEP) (BRASIL, 2012), sendo realizada após a aprovação pelo Comitê de Ética em Pesquisa (CEP) por meio da CAAE 28657820.8.0000.5298 e do parecer 3.913.312.

Adicionalmente, com a interpretação dos mesmos, foram elaboradas figura e tabelas com a finalidade de expor os resultados e confrontá-los com as informações disponíveis em bancos de dados eletrônicos específicos, como: Literatura Latino-Americana e do Caribe de Ciências da Saúde (LILACS), Scientific Eletronic Library Online (SciELO), U.S. National Library of Medicine (PubMed) e Google Acadêmico.

Para a busca dos trabalhos científicos, além dos bancos de dados eletrônicos, considerou-se também os seguintes aspectos; a) a combinação de descritores em ciências da saúde (DeCS) nos idiomas português, inglês e espanhol; b) palavras chave inerentes ao contexto abordado no título ou no resumo do trabalho; c) disponibilidade na íntegra; d) com exposição clara e concisa do assunto de interesse; e) estudos clínicos, de revisão bibliográfica ou sistemática e, f) pesquisas realizadas com seres humanos, exceto em indivíduos com faixa etária inferior a 18 anos. Destaca-se ainda que na busca dos trabalhos científicos aplicou-se os filtros review (revisão), free full text (texto completo grátis), data (2010-2020).

Em contrapartida, foram excluídos trabalhos científicos que não contemplaram as características descritas anteriormente, bem como, as pesquisas que apresentaram uma descrição metodológica não coerente (confusa), a fonte não era segura, pesquisas realizadas com animais e crianças e, aqueles que constavam duplicados.

\section{RESULTADOS E DISCUSSÃO}

Por meio da estratégia de busca de trabalhos científicos no banco de dados eletrônicos, obteve-se um total de 167 trabalhos científicos, entretanto, ao analisar criteriosamente cada um deles considerando os fatores de inclusão e exclusão descritos na metodologia, selecionou-se um total de 14 artigos para análise, revisão e discussão do presente trabalho científica (Figura 1).

No presente estudo, selecionou-se um total de 41 prontuários de pacientes vítimas de TCE grave que foram atendidos no Hospital de Urgência e Emergência, correspondendo ao período de maio a outubro de 2020, considerando os critérios de inclusão e exclusão (Tabela 1). A finalidade do setor de urgência e emergência de uma unidade hospitalar é acolher e diagnosticar o agravo, de uma maneira rápida com o objetivo de iniciar precocemente o tratamento adequado com a celeridade que cada caso clínico requer (MOLL AVS, 2015). 
Figura 1 - Fluxograma referente à seleção das publicações científicas no banco de dados eletrônicos,

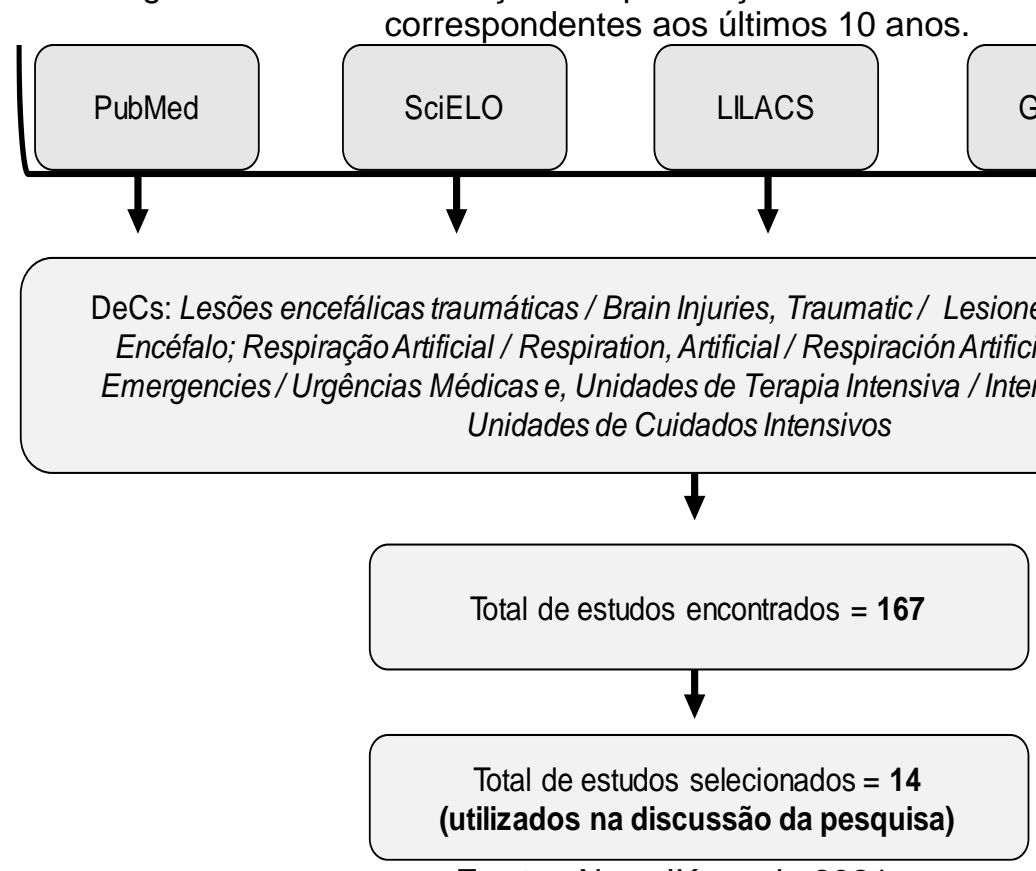

Fonte: Alves IK, et al., 2021.

Nota-se que houve relativamente poucas vítimas com o trauma mencionado no período de investigação, acredita-se que isso se deve ao fato da ocorrência da pandemia do SARS-COV-2, uma vez que muito se discutiu sobre as medidas de segurança em saúde pública, tendo em vista o seu contágio facilitado pelo contato próximo. Assim, determinou-se a necessidade de evitar as aglomerações, preservando a população de modo geral em casa. Nesse contexto, adotou-se o distanciamento social. Além disso, o indivíduo contaminado ou com suspeita de contaminação, estabeleceu-se um período de quarentena, por no mínimo 14 dias (SILVA MVS, et al., 2020). Sugerindo então, a diminuição dos acidentes que causam TCE.

Tabela 1 - Apresentação das variáveis observadas a partir da amostra de 41 prontuários médicos de vítimas de TCE grave.

\begin{tabular}{|c|c|}
\hline Variáveis & Valores médios \pm desvio padrão $(X \pm D P)$ \\
\hline \multicolumn{2}{|c|}{ Total da amostra $(n=41)$} \\
\hline \multicolumn{2}{|l|}{ Gênero } \\
\hline $\begin{array}{l}\text { Homens } \\
\text { Mulheres }\end{array}$ & $\begin{array}{l}N=32(78,05 \%) \\
N=9(21,95 \%)\end{array}$ \\
\hline \multicolumn{2}{|l|}{ Faixa etária } \\
\hline Faixa etária amostra total & $(37,29 \pm 14,70)$ \\
\hline Faixa etária dos homens & $(37,0 \pm 15,46)$ \\
\hline Faixa etária das mulheres & $(38,33 \pm 12,37)$ \\
\hline \multicolumn{2}{|l|}{ Fatores etiológicos dos TCE grave } \\
\hline Acidente com motocicleta & 24 casos $(58,54 \%)$ \\
\hline Acidente automobilístico & $8 \operatorname{casos}(19,5 \%)$ \\
\hline Queda de altura & 5 casos $(12,2 \%)$ \\
\hline Agressão física & 4 casos $(9,76 \%)$ \\
\hline \multicolumn{2}{|l|}{ Escore da Escala de Coma de Glasgow } \\
\hline Pontuação $=3,0$ pontos & 25 casos $(60,98 \%)$ \\
\hline Pontuação $=4,0$ pontos & 1 caso $(2,44 \%)$ \\
\hline Pontuação $=5.0$ pontos & 1 caso $(2,44 \%)$ \\
\hline Pontuação $=6.0$ pontos & 1 caso $(2,44 \%)$ \\
\hline Pontuação $=7.0$ pontos & $3 \operatorname{casos}(7,32 \%)$ \\
\hline Pontuação $=8.0$ pontos & 10 casos $(24,38 \%)$ \\
\hline \multicolumn{2}{|l|}{ Tempo de permanência na VMI } \\
\hline Permanência da amostra total $(n=41)$ & $9,70 \pm 8,25$ dias \\
\hline Variação do tempo de permanência na VMI $(n=41)$ & $2-29$ dias \\
\hline
\end{tabular}

Fonte: Alves IK, et al., 2021. 
Observou-se uma prevalência entre o gênero masculino com um total de 32 indivíduos, comparado com apenas 9 mulheres e, a faixa etária das vítimas de TCE grave demonstrou uma variação de 18 a 71 anos de idade e, a média da faixa etária correspondeu à $37,29 \pm 14,70$ anos de idade. Referente as causas do TCE grave, prevaleceu os acidentes com motocicleta (24 casos, $58,54 \%$ ), seguido de acidente automobilístico (8 casos, $19,5 \%$ ), queda de altura (5 casos, 12,2\%) e, os demais condizentes com a agressão física (4 casos, 9,76\%).

A maior incidência de TCE encontrada no gênero masculino tende a refletir as diferenças nas condições de exposição ao risco entre os gêneros, ou seja, a imaturidade e a sensação de impunidade e invulnerabilidade, associados ao uso de álcool e substâncias psicoativas, assim como o excesso de velocidade, atitudes radicais e violentas e o não uso dos equipamentos de segurança obrigatórios (desrespeito ao código de trânsito), é característica inerente à esse gênero (PADUA CS, et al., 2018; SOUSA MSR, et al., 2018; MAGALHÃES ALG, 2017; VIÉGAS MLC, et al., 2013).

O TCE é a principal causa de morte e sequelas na faixa etária de 1 a 44 anos de idade, que acomete principalmente homens e, referente as causas, os autores apontaram os acidentes envolvendo veículos automotivos, as quedas constituem o segundo grupo de lesões, sendo comum principalmente na população geriátrica, sendo que esse último sofrem importantes alterações fisiológicas advindas do processo do envelhecimento, como incoordenação motora, síncope, favorecendo as quedas (CONSTÂNCIO JF, et al., 2018; PADUA CS, et al., 2018; SOUSA MSR, et al., 2018).

No que diz respeito ao escore da Escala de Coma de Glasgow, constatou-se que houve um predomínio de 3 pontos (25 pacientes, 60,98\%), seguido daqueles que obtiveram uma pontuação de 8 pontos (10 casos, 24,38\%), os demais apresentaram uma pontuação de 4,0 (1 caso, 2,44\%); 5,0 (1 caso, 2,44\%); 6.0 ( 1 caso, 2,44\%) e 7.0 pontos ( 3 casos, 7,32 ).

O nível de consciência das vítimas após a ocorrência do trauma influência de forma significativa no seu prognóstico. Assim, é fundamental a classificação da gravidade através da Escala de Coma de Glasgow, a qual é um método que permite a avaliação neurológica com a qualificação do nível de consciência dos indivíduos após o trauma e, além disso, serve também como indicador da deterioração ou melhora da função cerebral à medida que se deve repetir a aplicação da escala ao longo do atendimento clínico (CONSTÂNCIO JF, et al., 2018; EBSERH, 2018).

Enfatiza-se que todo paciente vítima de TCE grave que apresenta uma Escala de Coma de Glasgow com pontuação igual ou inferior a 8 pontos deve ser intubado, dando ênfase aos cuidados hemodinâmicos e promovendo proteção de vias aéreas e manutenção da ventilação adequados (GENTILE JKA, et al., 2011; EBSERH, 2018).

Já em relação ao tempo de permanência e/ou dependência da VMI, observou-se que houve uma variação de 2 a 29 dias, sendo que o tempo médio foi de $9,70 \pm 8,25$ dias. Ao analisar a relação entre 0 tempo de permanência e a sobrevida ou não dos pacientes, constatou-se que aqueles que foram à óbito tiveram um tempo menor de permanência na VMI (6,76 $\pm 4,75$ dias) comparado com os que sobreviveram $(12,8 \pm 10,0$ dias $)$.

No estudo realizado por Constâncio JF, et al. (2018) constataram um tempo médio de internação de 11,5 \pm dias, valor superior ao encontrado no presente estudo. Já Padua CS, et al. (2018) encontraram uma média de internação de 12,68 \pm 9,67 dias, com no mínimo 2 e no máximo 53 dias, destacaram que o tempo elevado de permanência de internação hospitalar nesse público tem relação íntima com a gravidade do trauma e, sobretudo, com as possíveis complicações que são comuns.

Ressalta-se que a VMI constitui um importante mecanismo terapêutico nos pacientes com TCE grave, pois visa a proteção das vias aéreas, pela intubação orotraqueal e, permite a sedação e curarização, evitando os danos causados pela hipoxemia e hipercapnia. Em suma a VMI proporciona uma proteção em relação ao segundo trauma (causado por hipóxia ou hipercapnia) ou o "terceiro trauma" (que pode ocorrer após a hospitalização, quando por assistência ventilatória inadequada, surgem picos de elevação de PIC agravando o edema cerebral (PEREIRA DA, et al., 2018; GENTILE JKA, et al., 2011). 
Devido pacientes com TCE apresentarem recuperação lenta, a implementação da VMI por um período de tempo prolongado é necessária, ficando dessa forma vulneráveis ao desenvolvimento de complicações. A liberação da VMI envolve dois processos distintos: a) interrupção da VMI e a remoção do tubo orotraqueal (extubação). Entretanto, decidir se o paciente pode ou não tolerar a remoção do tubo orotraqueal é crucial, visto que um atraso ou a falha da extubação associam-se aos efeitos adversos e ao aumento da mortalidade (REIS HFC, et al., 2013).

Compete ainda enfatizar que entre os pacientes que sobreviveram $(n=20,48,78 \%)$, somente $7(35 \%)$ desse total foram submetidos à colocação da cânula de traqueostomia, sendo 5 do gênero masculino e os demais do gênero feminino, com média de faixa etária de 35,8 $\pm 17,62$ anos de idade, com o predomínio da pontuação na escala de coma de Glasgow equivalente à 3.0 pontos $(4,42 \pm 2,43$ pontos $)$ e, o tempo total médio de permanência e/ou dependência da VMI foi de 22,42 $\pm 5,74$. Desse modo, os pacientes com TCE grave submetidos à procedimento de traqueostomia apresentaram uma faixa etária maior $(35,8 \pm 17,62$ anos de idade), com pontuação na Escala de Coma de Glasgow menor (4,42 $\pm 2,43$ pontos) e com um tempo de dependência ou permanência na VMI maior (22,42 $\pm 5,74$ dias) (Tabela 2).

A necessidade de VMI, instabilidade hemodinâmica e alteração do reflexo pupilar são considerados como fatores que indicam mal prognóstico (MAGALHÃES ALG, 2017).

No estudo realizado por Khalili $\mathrm{H}$, et al. (2017) onde coletaram os dados de 152 pacientes com TCE com pontuação na Escala de Coma de Glasgow menor ou igual a 8 pontos, os pacientes que receberam a cânula de traqueostomia obtiveram resultados favoráveis, com menor taxa de pneumonia e tempo de internação reduzido. Assim, concluíram que a colocação da cânula de traqueostomia nesse grupo de pacientes, além de diminuir a permanência em hospitais, melhora o prognóstico dos mesmos.

Os pacientes com comprometimento neurológico persistente e grave frequentemente requerem o uso de VMI, no entanto, há pouca evidência disponível para orientar o manejo quanto a decisão de extubação desses pacientes. Assim, a equipe multiprofissional pode extubar precoce ou tardiamente ou ainda optarem pela introdução da cânula de traqueostomia. Ressalta-se que o momento ideal de extubação dos pacientes com TCE grave é desconhecido, bem como, não está claro se a traqueostomia primária é preferível a uma tentativa de extubação eletiva (MCCREDIE VA, et al., 2017).

Tabela 2 - Demonstração dos pacientes com TCE grave que sobreviveram foram submetidos ou não ao procedimento de introdução da cânula de traqueostomia em função da faixa etária, pontos na Escala de Coma de Glasgow e tempo de permanência na VMI.

\begin{tabular}{lccc}
\hline \multicolumn{1}{c}{ Amostra $(\mathbf{N}=\mathbf{4 1})$} & Faixa etária (anos) & ECG (pontos) & Tempo de VMI (dias) \\
\cline { 2 - 4 } & & $(\mathbf{X} \pm \mathbf{D P})$ & \\
\hline Sobreviventes $(\mathrm{N}=20,48,78 \%)$ & $32,8 \pm 13,03$ & $5,15 \pm 2,45$ & $12,8 \pm 10,00$ \\
$7 \mathrm{c} /$ traqueostomia $(35 \%)$ & $35,8 \pm 17,62$ & $4,42 \pm 2,43$ & $22,42 \pm 5,74$ \\
$13 \mathrm{~s}$ / traqueostomia $(65 \%)$ & $31,15 \pm 10,25$ & $5,53 \pm 2,47$ & $7,61 \pm 7,66$ \\
\hline
\end{tabular}

Fonte: Alves IK, et al., 2021.

$\mathrm{Na}$ análise dos dados coletados também foi possível averiguar que os pacientes com TCE grave que sobreviveram ( $\mathrm{N}=20,48,78 \%$ ) foram os que apresentaram os menores índices médios para faixa etária (32,8 $\pm 13,03$ anos de idade), e maiores médias de pontuação para a Escala de Coma de Glasgow (5,15 \pm 2,45 pontos) e tempo de permanência de VMI (12,8 $\pm 10,00$ dias), indicando que os pacientes com TCE grave que foram a óbito eram mais velhos e, tinham uma pontuação mais grave para a Escala de Coma de Glasgow e, consequentemente ficaram sujeitos ao menor tempo de VMI em virtude do óbito (Tabela 3).

Os estudos têm apresentado uma relação inversamente proporcional entre o número de mortes e a pontuação inicial na Escala de Coma de Glasgow, ou seja, quanto menor o valor do escore nessa escala, maior será o índice de mortalidade entre os indivíduos (CONSTÂNCIO CS, et al., 2018).

Os casos graves apresentam mortalidade que varia de $30-70 \%$ e, as vítimas que sobrevivem, muitas vezes, a recuperação é marcada por importantes e severas sequelas neurológicas e prejuízos na qualidade de vida (SOUSA MSR, et al., 2018) e, até mesmo as lesões cranianas tidas como pequenas podem resultar 
em sequelas, as vítimas de TCE com baixo escore na Escala de Coma de Glasgow, indicando maior gravidade, apresentam lesões que se apresentam com maior risco de morbimortalidade. Nesses pacientes a abordagem terapêutica deve ser de forma imediata, enfatizando os cuidados hemodinâmicos e suporte ventilatório adequados (MAGALHÃES ALG, 2017).

Tabela 3 - Apresentação dos dados dos pacientes com TCE grave (sobreviventes ou não) com a descrição da faixa etária, pontos na Escala de Coma de Glasgow e tempo de permanência na VMI.

\begin{tabular}{lccc}
\hline \multicolumn{1}{c}{ Amostra $(\mathbf{N}=\mathbf{4 1})$} & Faixa etária (anos) & ECG (pontos) & Tempo de VMI (dias) \\
\cline { 2 - 4 } & $32,8 \pm 13,03$ & $(\mathbf{X} \pm \mathbf{D P})$ \\
\hline Sobreviventes $(\mathrm{N}=20,48,78 \%)$ & $5,15 \pm 2,45$ & $12,8 \pm 10,00$ \\
Óbitos $(\mathrm{N}=21,51,22 \%)$ & $41,9 \pm 15,03$ & $4,19 \pm 1,93$ & $6,76 \pm 4,75$ \\
\hline
\end{tabular}

Fonte: Alves IK, et al., 2021.

\section{CONSIDERAÇÕES FINAIS}

Os pacientes com TCE grave, apresentam uma recuperação lenta e, geralmente necessitam de intubação orotraqueal, a qual ocorre por período de tempo prolongado, na maioria das vezes, predispondo as vítimas ao desenvolvimento de importantes complicações. Referente ao tempo de permanência da VMI, houve variação de dias entre pacientes com maior e menor pontuação da escala de coma de Glasgow, onde os que tiveram menor pontuação são os que em maioria permaneceram por menos tempo na ventilação, sendo indivíduos com maior gravidade do estado clínico geral. Nesse sentido, é fundamental que a equipe multiprofissional tenha amplo conhecimento sobre os aspectos inerentes a esse tipo de trauma, fornecendo atendimento as vítimas de TCE grave nos Hospitais de Urgência e Emergência.

\section{AGRADECIMENTOS}

Agradeço a minha família e amigos pelo apoio e compreensão durante a realização da minha residência, também a direção geral do Hospital de Urgência e Emergência que permitiu que essa pesquisa fosse desenvolvida na instituição, além de pessoas que direta ou indiretamente contribuíram para a realização da mesma, pois, são suficientemente meritórias para serem reconhecidas.

\section{REFERÊNCIAS}

1. BOER C, et al., Prehospital management of severe traumatic brain injury: concepts and ongoing controversies. Curr Opin Anaesthesiol. 2012; 25(5):556-62.

2. BOHMAN LE, et al., Medical management of compromised brain oxygen in patients with severe traumatic brain injury. Neurocrit Care. 2011; 14(3):361-9.

3. BRASIL. Ministério da Saúde. Conselho Nacional de Saúde. Resolução n. 466, de 12 de dezembro de 2012. Diretrizes e normas regulamentadoras de pesquisa envolvendo seres humanos. Brasília, 2012.

4. CONSTÂNCIO JF et al. Perfil clínico-epidemiológico de indivíduos com histórico de traumatismo cranioencefálico. Rev baiana enferm; 2018; 32:28235.

5. EBSERH POP. Reabilitação Fisioterapêutica nos Pacientes com diagnóstico de traumatismo craniencefálico Unidade de Reabilitação, Uberaba, 2018 - Versão 2.0. 20. Disponível em: http://www2.ebserh.gov.br/documents/147715/0/POP+fisioterapia+no+TCE+1.pdf/dc366d1d-457e-4156-ab2b1912774d8c45. Acesso em; 29/12/2020.

6. GALGANO M, et al., Traumatic Brain Injury: Current Treatment Strategies and Future Endeavors. Cell Transplantation. 2017:1118-1130.

7. GENTILE JKA et al. Condutas no paciente com trauma crânioencefálico. Rev Bras Clin Med. São Paulo, 2011; 9(1):74-82.

8. HADDAD SH, ARABI YM. Gerenciamento de cuidados críticos de lesão cerebral traumática grave em adultos. Scand J Trauma Resusc Emerg Med 20, 2012; 12.

9. HAMEL RN e SMOLIGA JM. Physical Activity Intolerance and Cardiorespiratory Dysfunction in Patients with Moderate-to-Severe Traumatic Brain Injury. Sports Med. 2019; 49(8):1183-1198.

10. JOVANOVIC B, et al. Risk factors for ventilator-associated pneumonia in patients with severe traumatic brain injury in a Serbian trauma centre, International Journal of Infectious Diseases,2015; 38:6-51.

11. JOVANOVIC B, et al. Twenty-Eight-Day Mortality of Blunt Traumatic Brain Injury and Co-Injuries Requiring Mechanical Ventilation. Med Princ Pract. 2016; 25(5):435-41.

12. KHALILI H. et al. Experience with Traumatic Brain Injury: Is Early Tracheostomy Associated with Better Prognosis? World Neurosurg. 2017; 103:88-93. 
13. KHELLAF A, et al. Avanços recentes em lesão cerebral traumática. J Neurol; 2019; 266:2878-2889.

14. MAAS A. Traumatic brain injury: Changing concepts and approaches. Chin J Traumatol. 2016; 19(1):3-6.

15. MAGALHÃES ALG. Perfil dos pacientes vítimas de traumatismo cranioencefálico atendidos no Hospital João XXIII em julho de 2016 e identificação dos fatores que influenciaram sua mortalidade. Dissertação (Mestrado em Neurociências) - Instituto de Ciências Biológicas. Universidade Federal de Minas Gerais, Belo Horizonte, $2017,39$.

16. MARCONI MA, LAKATOS EV. Fundamentos de Metodologia Científica. São Paulo: Atlas, 2017; 200p.

17. MCCREDIE VA, et al. Canadian Critical Care Trials Group. Airway Management Strategies for Brain-injured Patients Meeting Standard Criteria to Consider Extubation. A Prospective Cohort Study. Ann Am Thorac Soc, 2017; 14(1):8593.

18. MOLL AVS. Perfil do atendimento dos pacientes com traumatismo cranioencefálico nos hospitais de urgência e emergência sob gestão da Secretaria de Estado de Saúde do Rio de Janeiro. Dissertação (Mestrado) - Escola Nacional de Saúde Pública Sergio Arouca, Rio de Janeiro, 2015, 67.

19. PADUA CS et al. Perfil epidemiológico de pacientes com traumatismo crânio-encefálico (TCE) de uma unidade de terapia intensiva na cidade de Rio Branco-AC, Amazônia Ocidental. South American Journal of Basic Education, Technical and Technological, 2018; 5(1):125-136.

20. PEREIRA DA, et al., Efeitos da ventilação mecânica invasiva nos pacientes com traumatismo cranioencefálico na unidade de terapia intensiva: uma revisão da literatura. Revista Uniabeu, 2018; 11(28).

21. PICETTI E, et al. On the behalf of the ESICM NIC/ARF sections. Ventilatory strategies in patients with severe traumatic brain injury: the VENTILO Survey of the European Society of Intensive Care Medicine (ESICM). Crit Care. $2020 ; 17 ; 24(1): 158$.

22. PONTE FR et al. Vítimas de traumatismo cranioencefálico: perfil epidemiológico em uma unidade de terapia intensiva. Rev. enferm. UFPE on line, 2017; 1826-1834.

23. PROTHEROE RT, GWINNUTT CL. Early hospital care of severe traumatic brain injury. Anaesthesia. 2011; 66(11):1035-47.

24. REIS HFC, et al. Association between the rapid shallow breathing index and extubation success in patients with traumatic brain injury. Rev Bras Ter Intensiva. 2013; 25(3):212-7.

25. REIS HFC, et al. Extubation failure influences clinical and functional outcomes in patients with traumatic brain injury. J Bras Pneumol, 2013; 39(3):330-8.

26. REIS HFC, et al. Perfil clínico e funcional de pacientes com traumatismo crânio encefálico submetidos à traqueostomia. EFDeportes.com, Revista Digital. Buenos Aires, 2012; 171.

27. SCHIMER-MIKALSEN K, et al. Intracranial Pressure During Pressure Control and Pressure-Regulated Volume Control Ventilation in Patients with Traumatic Brain Injury: A Randomized Crossover trial. Neurocrit Care. 2016; 24(3):332-41.

28. SILVA MVS et al. O impacto do isolamento social na qualidade de vida dos idosos durante a pandemia por COVID19. Enfermagem Brasil, 2020; 19(4):34-41.

29. SILVA PE, et al. Neuromuscular electrophysiological disorders and muscle atrophy in mechanically-ventilated traumatic brain injury patients: New insights from a prospective observational study, Journal of Critical Care, 2018; 44:87-94.

30. SOUSA MSR, et al. Epidemiologia de traumatismo cranioencefálico em um hospital. Revista da Sociedade Brasileira de Clínica Médica, 2018; 16(1):21-24.

31. TRIMMEL H, et al. Intensivtherapie des Schädel-Hirn-Traumas beim Mehrfachverletzten: Entscheidungsfindung bei komplexer Pathophysiologie [Intensive care treatment of traumatic brain injury in multiple trauma patients: Decision making for complex pathophysiology]. Unfallchirurg. 2017; 120(9):739-744.

32. VELLA MA, et al. Acute Management of Traumatic Brain Injury. Surg Clin North Am. 2017; 97(5):1015-1030.

33. YOU W, et al. Intraventricular intracranial pressure monitoring improves the outcome of older adults with severe traumatic brain injury: an observational, prospective study. BMC Anesthesiol. 2016; 11;16(1):35. 